\title{
Unleashing the power of anti-tumor CD4+ T cells: novel insights into the curative mechanisms of chemoimmunotherapy for cancer
}

\author{
Tsadik G Habtetsion*, Gang Zhou
}

From 30th Annual Meeting and Associated Programs of the Society for Immunotherapy of Cancer (SITC 2015) National Harbor, MD, USA. 4-8 November 2015

\section{Background}

$\mathrm{CD}^{+} \mathrm{T}$ cells are critical mediators of anti-tumor immunity and orchestrate a broad range of immune responses against cancer. Previous studies from our lab and others have demonstrated that, adoptive transfer of tumor specific $\mathrm{CD} 4^{+} \mathrm{T}$ cells to lymphopenic hosts led to eradication of established tumors in mice models. Accumulating evidence from preclinical and clinical studies also suggest that $\mathrm{CD} 4^{+} \mathrm{T}$ cells in combination with chemotherapy can control tumor progression and recurrence. However, the molecular and cellular mechanisms by which tumor reactive $\mathrm{CD} 4^{+} \mathrm{T}$ cells eliminate a wide variety of tumors are not completely understood.

\section{Methods}

In this project, we set out to study the mechanisms underlying the therapeutic effect of chemo-immunotherapy in the form of cyclophosphamide (CTX) and tumor specific $\mathrm{CD}^{+} \mathrm{T}$ cells. Recent studies have revealed that combined effect of Th- 1 cytokines, IFN- $\gamma$ and TNF, drive both murine and human cancer cells in to senescence. In the present study we wanted to examine the specific roles of IFN- $\gamma$ and TNF- $\alpha$ in the setting of chemoimmunotherapy and the contribution of other immune cells in the tumor microenvironment to tumor rejection beside the donor $\mathrm{CD} 4^{+} \mathrm{T}$ cells.

\section{Results}

In a mouse model of colorectal cancer, we found that host-derived interferon gamma (IFN- $\gamma$ ) and expression of IFN- $\gamma \mathrm{R}$ are critical components of $\mathrm{CD} 4^{+} \mathrm{T}$ cell-mediated tumor rejection, whereas depletion of NK cells and macrophages separately did not compromise the therapeutic effect of the CTX and $\mathrm{CD} 4^{+} \mathrm{T}$ cells regimen. In addition, IFN- $\gamma$ appeared to drive tumor senescence and apoptosis in vivo, leading to a curative outcome. Furthermore, we analyzed the global metabolic profiling of tumor tissues at different time points before and after chemoimmunotherapy.

\section{Conclusions}

Our data suggests that $\mathrm{CD} 4^{+} \mathrm{T}$ cells reprogram the metabolic profiling in tumor, tipping the balance towards progressive tumor regression. These findings may provide new insights into mechanisms of tumor rejection by $\mathrm{CD}^{+} \mathrm{T}$ cells, and may help develop more effective anti-tumor strategies based on a rational combination of chemotherapy and anti-tumor $\mathrm{CD} 4^{+} \mathrm{T}$ cells.

Published: 4 November 2015

\section{doi:10.1186/2051-1426-3-S2-P18}

Cite this article as: Habtetsion and Zhou: Unleashing the power of antitumor CD4+ T cells: novel insights into the curative mechanisms of chemoimmunotherapy for cancer. Journal for ImmunoTherapy of Cancer 2015 3(Suppl 2):P18.

Georgia Regents University, Augusta, GA, USA 